\title{
PRACTICAL NUMBERS AMONG THE BINOMIAL COEFFICIENTS
}

\author{
PAOLO LEONETTI ${ }^{\dagger}$ AND CARLO SANNA ${ }^{\ddagger}$
}

\begin{abstract}
A practical number is a positive integer $n$ such that every positive integer less than $n$ can be written as a sum of distinct divisors of $n$. We prove that most of the binomial coefficients are practical numbers. Precisely, letting $f(n)$ denote the number of binomial coefficients $\left(\begin{array}{l}n \\ k\end{array}\right)$, with $0 \leq k \leq n$, that are not practical numbers, we show that

$$
f(n)<n^{1-(\log 2-\delta) / \log \log n}
$$

for all integers $n \in[3, x]$, but at most $O_{\gamma}\left(x^{1-(\delta-\gamma) / \log \log x}\right)$ exceptions, for all $x \geq 3$ and $0<\gamma<\delta<\log 2$. Furthermore, we prove that the central binomial coefficient $\left(\begin{array}{c}2 n \\ n\end{array}\right)$ is a practical number for all positive integers $n \leq x$ but at most $O\left(x^{0.88097}\right)$ exceptions. We also pose some questions on this topic.
\end{abstract}

\section{INTRODUCTION}

A practical number is a positive integer $n$ such that every positive integer less than $n$ can be written as a sum of distinct divisors of $n$. This property has been introduced by Srinivasan [19]. Estimates for the counting function of practical numbers have been given by Hausman-Shapiro [5], Tenenbaum [20], Margenstern [9], Saias [15], and finally Weingartner [21], who proved that there are asymptotically $C x / \log x$ practical numbers less than $x$, for some constant $C>0$, as previously conjectured by Margenstern [9]. On another direction, Melfi [11] proved that every positive even integer is the sum of two practical numbers, and that there are infinitely many triples $(n, n+2, n+4)$ of practical numbers. Also, Melfi [10] proved that in every Lucas sequence, satisfying some mild conditions, there are infinitely many practical numbers, and Sanna [17] gave a lower bound for their counting function.

In this work, we study the binomial coefficients which are also practical numbers. Our first result, informally, states that for almost all positive integers $n$

2010 Mathematics Subject Classification. Primary: 11B65, Secondary: 11N25.

Key words and phrases. binomial coefficient; central binomial coefficient; practical number.

$\dagger$ P. Leonetti is supported by the Austrian Science Fund (FWF), project F5512-N26.

$\ddagger$ C. Sanna is supported by a postdoctoral fellowship of INdAM and is a member of the INdAM group GNSAGA. 
there is a negligible amount of binomial coefficients $\left(\begin{array}{l}n \\ k\end{array}\right)$, with $0 \leq k \leq n$, which are not practical. Precisely, for each positive integer $n$, define

$$
f(n):=\#\left\{0 \leq k \leq n:\left(\begin{array}{l}
n \\
k
\end{array}\right) \text { is not a practical number }\right\} .
$$

Our first result is the following.

Theorem 1.1. For all $x \geq 3$ and $0<\gamma<\delta<\log 2$, we have

$$
f(n)<n^{1-(\log 2-\delta) / \log \log n}
$$

for all integers $n \in[3, x]$, but at most $O_{\gamma}\left(x^{1-(\delta-\gamma) / \log \log x}\right)$ exceptions.

As a consequence, we obtain that as $x \rightarrow+\infty$ almost all binomial coefficients $\left(\begin{array}{l}n \\ k\end{array}\right)$, with $0 \leq k \leq n \leq x$, are practical numbers.

Corollary 1.1. We have

$$
\sum_{n \leq x} f(n) \ll_{\varepsilon} x^{2-\left(\frac{1}{2} \log 2-\varepsilon\right) / \log \log x}
$$

for all $x \geq 3$ and $\varepsilon>0$.

Among the binomial coefficients, the central binomial coefficients $\left(\begin{array}{c}2 n \\ n\end{array}\right)$ are of great interest. In particular, several authors have studied their arithmetic and divisibility properties, see e.g. [1, 2, 14, 16, 18].

In this direction, our second result, again informally, states that almost all central binomial coefficients $\left(\begin{array}{c}2 n \\ n\end{array}\right)$ are practical numbers.

Theorem 1.2. For $x \geq 1$, the central binomial coefficient $\left(\begin{array}{c}2 n \\ n\end{array}\right)$ is a practical number for all positive integers $n \leq x$ but at most $O\left(x^{0.88097}\right)$ exceptions.

Probably, there are only finitely many positive integers $n$ such that $\left(\begin{array}{c}2 n \\ n\end{array}\right)$ is not a practical number. By a computer search, we found only three of them below $10^{6}$, namely $n=4,10,256$. However, proving the finiteness seems to be out of reach with actual techniques. Indeed, on the one hand, if $n$ is a power of 2 whose base 3 representation contains only the digits 0 and 1 , then it can be shown that $\left(\begin{array}{c}2 n \\ n\end{array}\right)$ is not a practical number (see Proposition 2.1 below). On the other hand, it is an open problem to establish whether there are finitely or infinitely many powers of 2 of this type $[4,6,8,12]$.

We conclude by leaving two open questions. Note that since $\left(\begin{array}{l}n \\ 0\end{array}\right)=\left(\begin{array}{l}n \\ n\end{array}\right)=1$, we have $0 \leq f(n) \leq n-1$ for all positive integers $n$. It is natural to ask when one of the equalities is satisfied.

Question 1.1. What are the positive integers $n$ such that $f(n)=0$ ?

Question 1.2. What are the positive integers $n$ such that $f(n)=n-1$ ? 
Regarding Question 1.1, if $f(n)=0$ then $n$ must be a power of 2, otherwise there would exist (see Lemma 2.4 below) an odd binomial coefficient $\left(\begin{array}{l}n \\ k\end{array}\right)$, with $0<k<n$, and since 1 is the only odd practical number, we would have $f(n)>0$. However, this is not a sufficient condition, since $f(8)=1$. Regarding Question 1.2, if $n=2^{k}-1$, for some positive integer $k$, then $f(n)=n-1$, because all the binomial coefficients $\left(\begin{array}{l}n \\ k\end{array}\right)$, with $0<k<n$, are odd (see Lemma 2.4 below) and greater than 1, and consequently they are not practical numbers. However, this is not a necessary condition, since $f(5)=4$.

Notation. We employ the Landau-Bachmann "Big Oh" notation $O$ and the associated Vinogradov symbol «. In particular, any dependence of the implied constants is indicated with subscripts. We write $p_{i}$ for the $i$ th prime number.

\section{Preliminaries}

This section is devoted to some preliminary results needed in the later proofs. We begin with some lemmas about practical numbers.

Lemma 2.1. If $m$ is a practical number and $n \leq 2 m$ is a positive integer, then mn is a practical number.

Proof. See [10, Lemma 4].

Lemma 2.2. If $d$ is a practical number and $n$ is a positive integer divisible by $d$ and having all prime factors not exceeding $2 d$, then $n$ is a practical number.

Proof. By hypothesis, there exist positive integers $q_{1}, \ldots, q_{k} \leq 2 d$ such that $n=d q_{1} \cdots q_{k}$. Then, using Lemma 2.1, it follows by induction that $d q_{1} \cdots q_{m}$ is practical for all $m=1, \ldots, k$. In particular, $n$ is practical.

Lemma 2.3. We have that $p_{1}^{a_{1}} \cdots p_{s}^{a_{s}}$ is a practical number, for all positive integers $a_{1}, \ldots, a_{s}$.

Proof. It follows easily by induction on $s$, using Lemma 2.1 and Bertrand's postulate $p_{i+1}<2 p_{i}$.

For each prime number $p$ and for each positive integer $n$, put

$$
T_{p}(n):=\#\left\{0 \leq k \leq n: p \nmid\left(\begin{array}{l}
n \\
k
\end{array}\right)\right\} .
$$

We have the following formula for $T_{p}(n)$.

Lemma 2.4. Let $p$ be a prime number and let

$$
n=\sum_{j=0}^{s} d_{j} p^{j}, \quad d_{0}, \ldots, d_{s} \in\{0, \ldots, p-1\}, \quad d_{s} \neq 0
$$


be the representation in base $p$ of the positive integer $n$. Then we have

$$
T_{p}(n)=\prod_{j=0}^{s}\left(d_{j}+1\right)
$$

Proof. See [3, Theorem 2].

For each prime number $p$, let us define

$$
\omega_{p}:=\frac{\log ((p+1) / 2)}{\log p} .
$$

The quantity $\omega_{p}$ appears in the following upper bound for $T_{p}(n)$.

Lemma 2.5. Let $p$ be a prime number and $f i x \in \in(0,1 / 2)$. Then, for all $x \geq 1$, we have

$$
T_{p}(n)<n^{\omega_{p}+\varepsilon}
$$

for all positive integers $n \leq x$ but at most $O\left(p^{3} x^{1-\varepsilon}\right)$ exceptions.

Proof. For $x \geq 1$, let $k$ be the smallest integer such that $x<p^{k}$. Clearly, we have

$$
\begin{aligned}
E(x) & :=\#\left\{n \leq x: T_{p}(n) \geq n^{\omega_{p}+\varepsilon}\right\} \\
& \leq \sum_{j=1}^{k} \#\left\{p^{j-1} \leq n<p^{j}: T_{p}(n) \geq p^{(j-1)\left(\omega_{p}+\varepsilon\right)}\right\} .
\end{aligned}
$$

Moreover, thanks to Lemma 2.4, we have

$$
\sum_{p^{j-1}<n \leq p^{j}} T_{p}(n) \leq \sum_{0 \leq d_{0}, \ldots, d_{j-1}} \prod_{i=0}^{j-1}\left(d_{i}+1\right)=\left(\sum_{d=0}^{p-1}(d+1)\right)^{j}=\left(\frac{p(p+1)}{2}\right)^{j},
$$

and consequently

$$
\begin{aligned}
\#\left\{p^{j-1}\right. & \left.\leq n<p^{j}: T_{p}(n) \geq p^{(j-1)\left(\omega_{p}+\varepsilon\right)}\right\} \leq \frac{1}{p^{(j-1)\left(\omega_{p}+\varepsilon\right)}} \sum_{p^{j-1}<n \leq p^{j}} T_{p}(n) \\
& \leq \frac{1}{p^{(j-1)\left(\omega_{p}+\varepsilon\right)}}\left(\frac{p(p+1)}{2}\right)^{j}=\frac{p(p+1)}{2} p^{(1-\varepsilon)(j-1)}<p^{2+(1-\varepsilon)(j-1)},
\end{aligned}
$$

for all positive integers $j$. Therefore, putting together (1) and (2), and using that $\varepsilon<1 / 2$, we obtain

$$
E(x)<\sum_{j=1}^{k} p^{2+(1-\varepsilon)(j-1)} \ll p^{2+(1-\varepsilon) k} \leq p^{2+(1-\varepsilon)(\log x / \log p+1)}<p^{3} x^{1-\varepsilon},
$$

as desired. 
Remark 2.1. The constant $1 / 2$ in the statement of Lemma 2.5 has no particular importance, it is only needed to justify the $\ll$ in $(3)$. Any other real number less than 1 would be fine.

For all $x \geq 1$, let $\kappa(x)$ be the smallest integer $k \geq 1$ such that $p_{1} \cdots p_{k} \geq x$.

Lemma 2.6. We have

as $x \rightarrow \infty$.

$$
\kappa(x) \sim \frac{\log x}{\log \log x} \quad \text { and } \quad p_{\kappa(x)} \sim \log x,
$$

Proof. As a well-known consequence of the Prime Number Theorem, we have

$$
\log \left(p_{1} \cdots p_{k}\right) \sim p_{k} \sim k \log k
$$

as $k \rightarrow+\infty$. Since

$$
\log \left(p_{1} \cdots p_{\kappa(x)-1}\right)<\log x \leq \log \left(p_{1} \cdots p_{\kappa(x)}\right),
$$

and $\kappa(x) \rightarrow+\infty$ as $x \rightarrow+\infty$, by (4) we obtain

$$
p_{\kappa(x)} \sim \kappa(x) \log \kappa(x) \sim \log x,
$$

which in turn implies

$$
\kappa(x) \sim \frac{\kappa(x) \log \kappa(x)}{\log \kappa(x)+\log \log \kappa(x)} \sim \frac{\log x}{\log \log x},
$$

as desired.

For every prime number $p$ and every positive integer $n$, let $\beta_{p}(n)$ be the $p$-adic valuation of the central binomial coefficient $\left(\begin{array}{c}2 n \\ n\end{array}\right)$.

Lemma 2.7. For each prime $p$ and all positive integers $n$, we have that $\beta_{p}(n)$ is equal to the number of digits of $n$ in base $p$ which are greater than $(p-1) / 2$.

Proof. The claim is a straightforward consequence of a theorem of Kummer [7] which says that, for positive integers $m, n$, the $p$-adic valuation of $\left(\begin{array}{c}m+n \\ n\end{array}\right)$ is equal to the number of carries in the addition $m+n$ done in base $p$.

Proposition 2.1. If $n$ is a power of 2 and if all the digits of $n$ in base 3 are equal to 0 or 1 , then $\left(\begin{array}{c}2 n \\ n\end{array}\right)$ is not a practical number.

Proof. It follows by Lemma 2.7 that $\beta_{2}(n)=1$ and $\beta_{3}(n)=0$, that is, $\left(\begin{array}{c}2 n \\ n\end{array}\right)$ is an integer of the form $12 k \pm 2$. However, it is known that, other than 1 and 2 , every practical number is divisible by 4 or 6 , see [19].

We will make use of the following result of probability theory. 
Lemma 2.8. Let $X$ be a random variable following a binomial distribution with $j$ trials and probability of success $\alpha$. Then

$$
\mathbb{P}[X \leq(\alpha-\varepsilon) j] \leq e^{-2 \varepsilon^{2} j}
$$

for all $\varepsilon>0$.

Proof. See [13, Theorem 1].

For each prime number $p$, let us define

$$
\alpha_{p}:=\frac{1}{p}\left\lceil\frac{p-1}{2}\right\rceil,
$$

so that $\alpha_{p}$ is the probability that a random digit in base $p$ is greater than $(p-1) / 2$.

Lemma 2.9. Let $p$ be a prime number and fix $\varepsilon \in(0,1 / 2)$. Then, for all $x \geq 1$, we have

$$
\beta_{p}(n)>\left(\alpha_{p}-\varepsilon\right) \frac{\log n}{\log p}
$$

for all positive integers $n \leq x$ but at most $O\left(p x^{1-2 \varepsilon^{2} / \log p}\right)$ exceptions.

Proof. For $x \geq 1$, let $k$ be the smallest integer such that $x<p^{k}$. Clearly, we have

$$
\begin{aligned}
E(x) & :=\#\left\{n \leq x: \beta_{p}(n) \leq\left(\alpha_{p}-\varepsilon\right) \frac{\log n}{\log p}\right\} \\
& \leq \sum_{j=1}^{k} \#\left\{p^{j-1} \leq n<p^{j}: \beta_{p}(n) \leq\left(\alpha_{p}-\varepsilon\right) j\right\} \\
& \leq \sum_{j=1}^{k} \#\left\{0 \leq n<p^{j}: \beta_{p}(n) \leq\left(\alpha_{p}-\varepsilon\right) j\right\} .
\end{aligned}
$$

Given an integer $j \geq 1$, let us for a moment consider $n$ as a random variable uniformly distributed in $\left\{0, \ldots, p^{j}-1\right\}$. Then, the digits of $n$ in base $p$ are $j$ independent random variables uniformly distributed in $\{0, \ldots, p-1\}$. Hence, as a consequence of Lemma 2.7, we obtain that $\beta_{p}(n)$ follows a binomial distribution with $j$ trials and probability of success $\alpha_{p}$. In turn, Lemma 2.8 yields

$$
\#\left\{0 \leq n<p^{j}: \beta_{p}(n) \leq\left(\alpha_{p}-\varepsilon\right) j\right\} \leq p^{j} e^{-2 \varepsilon^{2} j} .
$$

Therefore, putting together (5) and (6), and using that $\varepsilon<1 / 2$, we get

$$
E(x) \leq \sum_{j=1}^{k} p^{j} e^{-2 \varepsilon^{2} j} \ll\left(p e^{-2 \varepsilon^{2}}\right)^{k} \leq\left(p e^{-2 \varepsilon^{2}}\right)^{\log x / \log p+1}<p x^{1-2 \varepsilon^{2} / \log p},
$$

as desired. 
Remark 2.2. The constant $1 / 2$ in the statement of Lemma 2.9 has no particular importance, it is only needed to justify the $\ll$ in $(7)$. Any other real number less than $\left(\frac{1}{2} \log 2\right)^{1 / 2}$ would be fine.

\section{Proof of Theorem 1.1}

Assume $x \geq 3$ sufficiently large, and put

$$
\varepsilon:=\frac{\delta-\gamma}{\log \log x}+\frac{4 \log \log x}{\log x} \in(0,1 / 2) .
$$

Let $n$ be a positive integer. By Lemma 2.3 and by the definition of $\kappa(n)$, we know that $p_{1} \cdots p_{\kappa(n)}$ is a practical number greater than or equal to $n$. Since all the prime factors of $\left(\begin{array}{l}n \\ k\end{array}\right)$ are not exceeding $n$, Lemma 2.2 tell us that if $p_{1} \cdots p_{\kappa(n)}$ divides $\left(\begin{array}{l}n \\ k\end{array}\right)$ then $\left(\begin{array}{l}n \\ k\end{array}\right)$ is practical. Consequently, we have

$$
f(n) \leq \#\left\{0 \leq k \leq n: p_{1} \cdots p_{\kappa(n)} \nmid\left(\begin{array}{l}
n \\
k
\end{array}\right)\right\} \leq \sum_{j=1}^{\kappa(n)} T_{p_{j}}(n) .
$$

Therefore, it follows from Lemma 2.5 that

$$
f(n)<\sum_{j=1}^{\kappa(n)} n^{\omega_{p_{j}}+\varepsilon}
$$

for all positive integers $n \leq x$ but at most

$$
\ll \sum_{j=1}^{\kappa(x)} p_{j}^{3} x^{1-\varepsilon} \ll p_{\kappa(x)}^{4} x^{1-\varepsilon} \ll(\log x)^{4} x^{1-\varepsilon}=x^{1-(\delta-\gamma) / \log \log x}
$$

exceptions, where we also used Lemma 2.6.

Suppose that $n$ satisfies (8). Since $\omega_{p}$ is a monotone increasing function of $p$, we get that

$$
f(n)<\kappa(n) n^{\omega_{p_{\kappa(n)}}+\varepsilon}=n^{\omega_{p_{\kappa(n)}}+\log \kappa(n) / \log n+\varepsilon} .
$$

Moreover, for $n \gg_{\gamma} 1$ we have

$$
\omega_{p_{\kappa(n)}}<1-\frac{\log 2}{\log p_{\kappa(n)}}+\frac{1}{p_{\kappa(n)} \log p_{\kappa(n)}}<1-\frac{\log 2-\gamma / 4}{\log \log n},
$$

and

$$
\frac{\log \kappa(n)}{\log n}<\frac{\gamma / 4}{\log \log n},
$$

where we used Lemma 2.6. Furthermore, since $n \leq x$, we have

$$
\varepsilon<\frac{\delta-\gamma / 2}{\log \log n}
$$


Consequently, putting together (10), (11), and (12), we obtain

$$
\omega_{p_{\kappa(n)}}+\frac{\log \kappa(n)}{\log n}+\varepsilon<1-\frac{\log 2-\delta}{\log \log n},
$$

which, inserted into (9), gives

$$
f(n)<n^{1-(\log 2-\delta) / \log \log n}
$$

as desired. The proof is complete.

\section{Proof of Corollary 1.1}

Obviously, we can assume $\varepsilon<\frac{1}{2} \log 2$. Put $\gamma:=2 \varepsilon$ and $\delta:=\frac{1}{2} \log 2+\varepsilon$, so that $0<\gamma<\delta<\log 2$. For all $x \geq 3$, let $\mathcal{E}(x)$ be the set of exceptional $n \leq x$ of Theorem 1.1. Then we have

$$
\begin{aligned}
\sum_{n \leq x} f(n) & =\sum_{n \notin \mathcal{E}(x)} f(n)+\sum_{n \in \mathcal{E}(x)} f(n)<\sum_{n \leq x} n^{1-(\log 2-\delta) / \log \log n}+\# \mathcal{E}(x) x \\
& \ll_{\varepsilon} x^{2-(\log 2-\delta) / \log \log x}+x^{2-(\delta-\gamma) / \log \log x} \ll x^{2-\left(\frac{1}{2} \log 2-\varepsilon\right) / \log \log x},
\end{aligned}
$$

as claimed.

\section{Proof of Theorem 1.2}

For the sake of notation, put

$$
s:=16, \quad \eta_{s}:=\frac{\sum_{i=1}^{s} \alpha_{p_{i}}-1}{\sum_{i=1}^{s} \sqrt{\log p_{i}}}, \quad \varepsilon_{j}:=\eta_{s} \sqrt{\log p_{j}},
$$

for $j=1, \ldots, s$. A computation shows that $\varepsilon_{j} \in(0,1 / 2)$ for $j=1, \ldots, s$.

For $x \geq 1$, it follows from Lemma 2.9 that

$$
\sum_{j=1}^{s} \beta_{p_{j}}(n) \log p_{j}>\sum_{j=1}^{s}\left(\alpha_{p_{j}}-\varepsilon_{j}\right) \log n=\log n,
$$

for all positive integers $n \leq x$, but at most

$$
\ll \sum_{j=1}^{s} p_{j} x^{1-2 \varepsilon_{j}^{2} / \log p_{j}} \ll x^{1-2 \eta_{s}^{2}}<x^{0.88097}
$$

exceptions. Suppose that $n$ is a positive integer satisfying (13). Then,

$$
d:=\prod_{j=1}^{s} p_{j}^{\beta_{p_{j}}(n)}>n
$$

Also, by Lemma 2.3 we have that $d$ is a practical number, and by the definition of $\beta_{p_{j}}(n)$ we have that $d$ divides $\left(\begin{array}{c}2 n \\ n\end{array}\right)$. Moreover, since all the prime factors of 
$\left(\begin{array}{c}2 n \\ n\end{array}\right)$ are not exceeding $2 d$, Lemma 2.2 yields that $\left(\begin{array}{c}2 n \\ n\end{array}\right)$ is practical. The proof is complete.

Remark 5.1. A comment is in order to explain the choice of the parameters $\varepsilon_{j}$ in the proof of Theorem 1.2. Given a positive integer $s$, one could fix some prime numbers $q_{1}<\cdots<q_{s}$ and some real numbers $\varepsilon_{1}, \ldots, \varepsilon_{s} \in(0,1 / 2)$ such that $q_{1} \cdots q_{s}$ is a practical number and $\sum_{j=1}^{s}\left(\alpha_{q_{j}}-\varepsilon_{j}\right) \geq 1$. Everything would proceed similarly, with an estimate of the number of exceptions given by

$$
O\left(x^{\max \left\{1-2 \varepsilon_{1}^{2} / \log q_{1}, \ldots, 1-2 \varepsilon_{s}^{2} / \log q_{s}\right\}}\right) .
$$

To minimize the exponent of $x$, the optimal choice for $\varepsilon_{j}$ is

$$
\varepsilon_{j}=\eta_{s}\left(q_{1}, \ldots, q_{s}\right) \sqrt{\log q_{j}}, \quad \eta_{s}\left(q_{1}, \ldots, q_{s}\right):=\frac{\sum_{i=1}^{s} \alpha_{q_{i}}-1}{\sum_{i=1}^{s} \sqrt{\log q_{j}}},
$$

for $j=1, \ldots, s$, which gives the estimate

$$
O\left(x^{1-2 \eta_{s}\left(q_{1}, \ldots, q_{s}\right)^{2}}\right) .
$$

Since $\alpha_{p}=\frac{1}{2}+O\left(\frac{1}{p}\right)$ for each prime number $p$, we get that $\eta_{s}\left(q_{1}, \ldots, q_{s}\right)$ is maximized when $q_{j}=p_{j}$, for $j=1, \ldots, s$, and that $\eta_{s}\left(p_{1}, \ldots, p_{s}\right) \rightarrow 0$ as $s \rightarrow+\infty$. Lastly, some numeratical computations verify that the maximum of $\eta_{s}\left(p_{1}, \ldots, p_{s}\right)$ is reached for $s=16$.

\section{REFERENCES}

1. D. Berend and J. E. Harmse, On some arithmetical properties of middle binomial coefficients, Acta Arith. 84 (1998), no. 1, 31-41.

2. P. Erdős, R. L. Graham, I. Z. Ruzsa, and E. G. Straus, On the prime factors of $\left(\begin{array}{c}2 n \\ n\end{array}\right)$, Math. Comp. 29 (1975), 83-92, Collection of articles dedicated to Derrick Henry Lehmer on the occasion of his seventieth birthday.

3. N. J. Fine, Binomial coefficients modulo a prime, Amer. Math. Monthly 54 (1947), 589-592.

4. H. Gupta, Powers of 2 and sums of distinct powers of 3, Univ. Beograd. Publ. Elektrotehn. Fak. Ser. Mat. Fiz. (1978), no. 602-633, 151-158 (1979).

5. M. Hausman and H. N. Shapiro, On practical numbers, Comm. Pure Appl. Math. 37 (1984), no. $5,705-713$.

6. R. E. Kennedy and C. Cooper, A generalization of a result by Narkiewicz concerning large digits of powers, Univ. Beograd. Publ. Elektrotehn. Fak. Ser. Mat. 11 (2000), 36-40 (2001).

7. E. E. Kummer, Über die Ergänzungssätze zu den allgemeinen Reciprocitätsgesetzen, J. Reine Angew. Math. 44 (1852), 93-146.

8. J. C. Lagarias, Ternary expansions of powers of 2, J. Lond. Math. Soc. (2) 79 (2009), no. 3, 562-588.

9. M. Margenstern, Les nombres pratiques: théorie, observations et conjectures, J. Number Theory 37 (1991), no. 1, 1-36. 
10. G. Melfi, A survey on practical numbers, Rend. Sem. Mat. Univ. Politec. Torino 53 (1995), no. 4, 347-359, Number theory, II (Rome, 1995).

11. G. Melfi, On two conjectures about practical numbers, J. Number Theory 56 (1996), no. 1, 205-210.

12. W. Narkiewicz, A note on a paper of H. Gupta concerning powers of two and three: "Powers of 2 and sums of distinct powers of 3 " [Univ. Beograd. Publ. Elektrotehn. Fak. Ser. Mat. Fiz. No. 602-633 (1978), 151-158 (1979); MR 81g:10016], Univ. Beograd. Publ. Elektrotehn. Fak. Ser. Mat. Fiz. (1980), no. 678-715, 173-174 (1981).

13. M. Okamoto, Some inequalities relating to the partial sum of binomial probabilities, Ann. Inst. Statist. Math. 10 (1958), 29-35.

14. C. Pomerance, Divisors of the middle binomial coefficient, Amer. Math. Monthly 122 (2015), no. 7, 636-644.

15. E. Saias, Entiers à diviseurs denses. I, J. Number Theory 62 (1997), no. 1, 163-191.

16. C. Sanna, Central binomial coefficients divisible by or coprime to their indices, Int. J. Number Theory 14 (2018), no. 4, 1135-1141.

17. C. Sanna, Practical numbers in Lucas sequences, Quaest. Math. (in press), https://doi.org/10.2989/16073606.2018.1502697.

18. A. Sárközy, On divisors of binomial coefficients. I, J. Number Theory 20 (1985), no. 1, 70-80.

19. A. K. Srinivasan, Practical numbers, Current Sci. 17 (1948), 179-180.

20. G. Tenenbaum, Sur un problème de crible et ses applications, Ann. Sci. École Norm. Sup. (4) 19 (1986), no. 1, 1-30.

21. A. Weingartner, Practical numbers and the distribution of divisors, Q. J. Math. 66 (2015), no. $2,743-758$.

Graz University of Technology, Institute of Analysis and Number Theory, Graz, Austria

E-mail address: leonetti.paolo@gmail.com

Università degli Studi di Genova, Department of Mathematics, Genova, Italy

E-mail address: carlo.sanna.dev@gmail.com 